\title{
Exploring the solar system: the view of planetary surfaces with VIS/IR remote sensing methods
}

\section{Gabriele Arnold}

Gabriele E. Arnold, "Exploring the solar system: the view of planetary surfaces with VIS/IR remote sensing methods," Proc. SPIE 8154, Infrared Remote Sensing and Instrumentation XIX, 815402 (17 September 2011); doi: 10.1117/12.897759

SPIE Event: SPIE Optical Engineering + Applications, 2011, San Diego, California, United States 


\title{
Exploring the solar system: the view of planetary surfaces with VIS/IR remote sensing methods
}

\author{
Gabriele E. Arnold \\ Deutsches Zentrum für Luft- und Raumfahrt e.V. (DLR), Rutherfordstrasse 2, 12489 Berlin, \\ Germany \\ * Gabriele.Arnold@dlr.de; phone +49-3067055370; fax + 49-3067055303
}

\begin{abstract}
The structure of planetary surfaces unveils basic formation processes and evolution lines of different objects in the solar system, and often the view on the top of a planet is the only available information about it. Advanced remote sensing technologies on deep space missions are aimed at accessing a maximum of relevant data to characterize a planetary object holistically. This approach requires concert strategies in planetary and engineering science. In this framework VIS/IR spectroscopic remote sensing methods are key technologies for imaging planetary atmospheres and surfaces, for studying their composition, texture, structure and dynamics. Basing on these analyses it succeeds to observe the single objects in more global geo-scientific content. The paper focuses on main geo-scientific output coming from spectroscopic studies of planetary surfaces in conjunction with their interiors, atmospheres, and the interplanetary space. It summarizes selected results of spectral studies onboard of the ESA deep space missions BepiColombo, Venus Express, Mars Express, and Rosetta. The corresponding spectral instruments are introduced. The complex conflation of special knowledge of the disciplines planetology, optical and IR measuring techniques, and space flight engineering is demonstrated in several examples. Finally, the paper gives an outlook of current developments for spectral studies in planned missions, and sums up some of the driving questions in planetary science.
\end{abstract}

Keywords: Planetary physics, planetary remote sensing, spectroscopy, planetary surfaces, surface composition, planetology

\section{INTRODUCTION}

Spacecraft studies of planetary bodies have increasingly contributed to our knowledge about the single objects over the last decades. The planetary surface provides the link between the interior of the planet and its atmosphere or the interplanetary space. The geological, physical and chemical structure reveals key information about formation and evolution processes in the solar system. Advanced planetary remote sensing includes 3-D imaging techniques with high spatial resolution, global monitoring, and multiple coverage strategies for object recognition and characterization. Stratigraphic techniques basing on photogeologic studies allow reading the record left by geologic processes. The principle of these analyses is superposition and transaction. For the surfaces of terrestrial bodies, the relative chronology can be studied by crater size-frequency distributions (SFD). ${ }^{13}$ The chronology of major geologic units opens up a way to reconstruct the history of the single planetary body. Comparative analyses of these evolution paths offer the capability for planetologic studies, considering general formation mechanisms in the solar system. In addition to the object recognition on planetary surfaces, different thematic and dynamic information is required to verify and analyze the original sequences. Among the various methods, visual and infrared (VIS/IR) spectroscopic remote sensing is a key technology to investigate planetary surfaces, and the adjacent atmosphere for studying their composition, texture, structure and dynamics. During the last twenty years high-resolution (spatial) multispectral imaging systems for photogeologic surface mapping and multi-hyperspectral sensors for thematic mapping have been developed to operate together for planetologic applications on ESA's deep space missions. Planetary spectrometers which were successfully developed, applied and operated on different European planetary projects can be divides into three groups: imaging spectrometers for the 0.25 to $5 \mu \mathrm{m}$ range $\mathrm{e}^{34,35,36,37}$, imaging spectrometers in the $7-14 \mu \mathrm{m}$ region $^{6,8,9,53}$, and interferometers

Infrared Remote Sensing and Instrumentation XIX, edited by Marija Strojnik, Gonzalo Paez,

Proc. of SPIE Vol. 8154, 815402 - @ 2011 SPIE · CCC code: 0277-786X/11/\$18 · doi: 10.1117/12.897759 
from 1.25 up to $45 \mu \mathrm{m}^{4,5,41,42,54-56}$. Covering the wavelength range from the UV/VIS up to the mid-infrared region, these hyperspectral systems use the reflected sunlight or the thermal emission from the different objects. Depending on the spectral radiance and on the single scientific questions to be answered, the design of planetary hyperspectral systems has to be optimized in terms of spatial, spectral, radiometric, and temporal resolution, resulting in specific instrumental requirements. The technical implementation of these requirements is often limited by extreme environmental conditions, restricted resources in volume, mass and power, long operation distances, autonomy requests, and telemetry conditions. Spacecraft VIS/IR remote sensing of planetary surfaces requires a close and common approach of planetary and engineering sciences directed to match a maximum of scientific requests and space flight qualified instrumental solutions.

\section{PERFORMANCE OF PLANETARY HYPERSPECTRAL SYSTEMS}

The spectral radiance of airless planetary objects can be estimated knowing the solar irradiance, the distance of the object from the sun, its spectral reflectance, emissivity and the surface temperature. Figure 1 shows the spectral radiance of chosen airless objects in the planetary system. The radiance is composed of two parts. The VIS and NIR range is dominated by the surface reflection of solar irradiance, while thermal emission determines the radiance coming from the planetary body in the mid- and thermal infrared range. Assuming the surface to irradiate like a black body at surface temperature, the estimation provides the radiance maximum position controlled by Wien's displacement law.

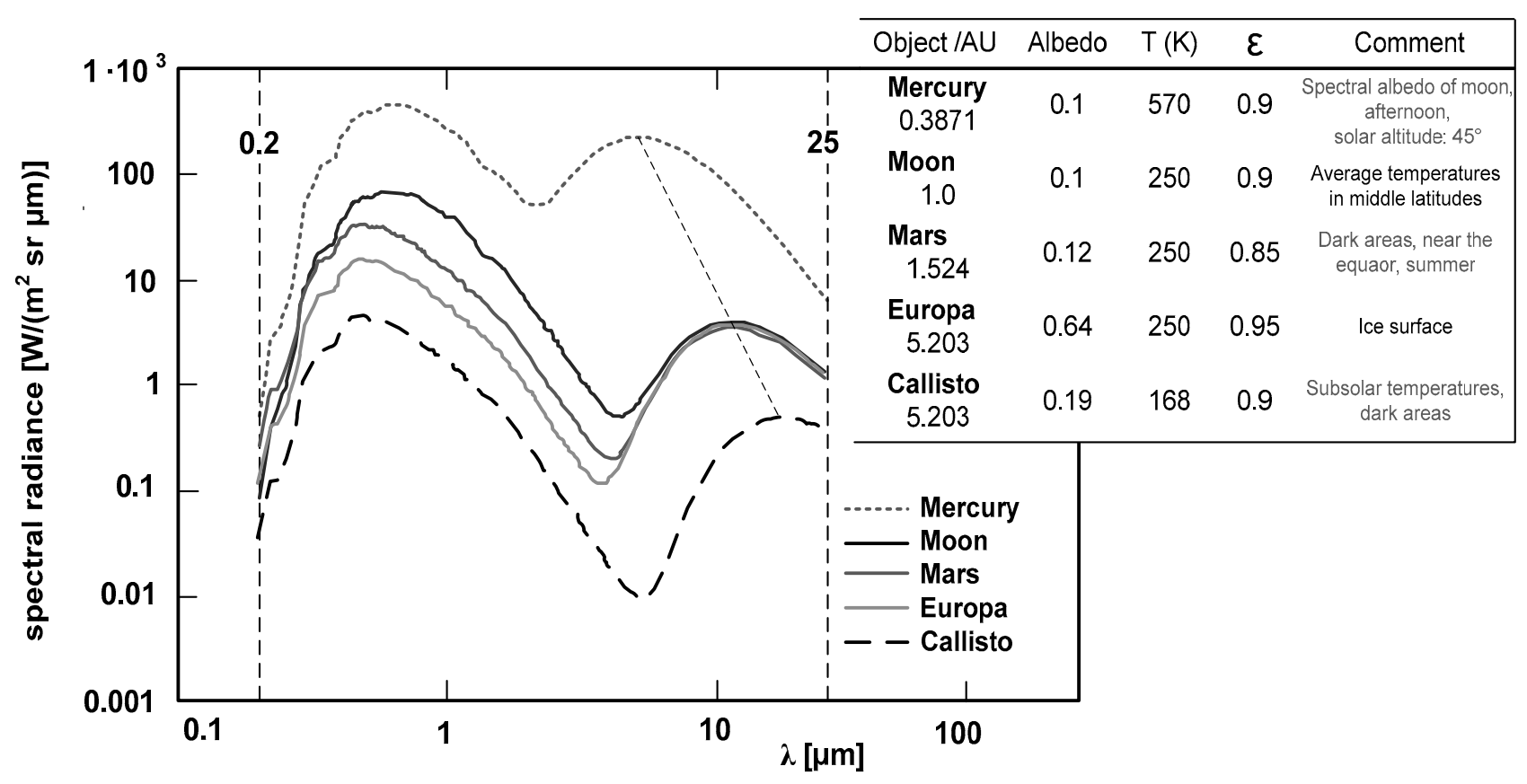

Figure 1. Spectral radiance of airless planetary objects. The sequence of solid lines from top to bottom corresponds with the legend.

Thermal radiation of a planetary surface depends on the kinetic temperature of the surface and the surface emissivity. The boundary between both radiation components shifts toward shorter wavelength with increasing surface temperature, defining the observation conditions for a particular object. Both wavelength ranges (the VIS/NIR and thermal infrared (TIR) part of the spectrum) are diagnostic for the surface composition analysis of rocky bodies in the solar system. Whereas the VIS/NIR region provides information about absorption bands caused by electronic processes and/or overtone and combination tone bands of lattice vibrations, the mid-infrared reveals the spectral features of the fundamental vibrations bands of solid surface materials. Additionally, particle size affects the reflectance or emissivity spectra and the thermal inertia, providing information about textural parameters of the soil. ${ }^{3}$ 


\subsection{Planetary imaging spectrometers}

\section{Visible and InfraRed Thermal Imaging Spectrometer (VIRTIS)}

The VIRTIS instrument was developed to observe planetary objects in the range between 0.28 and $5.1 \mu \mathrm{m}$. VIRTIS stands for Visible and InfraRed Thermal Imaging Spectrometer. This instrument was developed to study the comet $67 \mathrm{P} /$ Churyumov-Gerasimenko on board the ESA cornerstone mission ROSETTA. ${ }^{73,75}$ VIRTIS-ROSETTA is a development of an international consortium between Italy, France, and Germany under leadership of Angioletta Coradini (Italy). VIRTIS combines a two-channel imaging spectrometer (VIRTIS-M) for surface mapping with spectral high resolution Echelle spectrometer (VIRTIS-H). The two VIRTIS-M channels (VIS and IR) are devoted to spectral mapping (Mapper optical subsystem -M) at moderate spectral resolution in the spectral range from 0.28 to $5.13 \mu \mathrm{m}$. The third channel is devoted to spectroscopy (High resolution optical subsystem -H) in the spectral range from about 2 to $5 \mu \mathrm{m}$. VIRTIS-M for ROSETTA was built by Galileo Avionica Florence (Italy), VIRTIS-H by Paris Observatory (France) and the electronic module and data handling unit by Kayser-Threde for hardware and DLR for software and management (Germany).

Table1. Instrumental parameters of the three VIRTIS data channels (based on Table 1 in [70]).

\begin{tabular}{lccc}
\hline & VIRTIS-M Infrared & VIRTIS-M Visible & VIRTIS-H \\
\hline Spectral range $(\mu \mathrm{m})$ & $1.02-5.13$ & $0.28-1.10$ & $1.84-5.00$ \\
Spectral resolution $\lambda / \Delta \lambda$ & $\sim 200$ & $\sim 200$ & $\sim 1200$ \\
Spectral sampling $(\mathrm{nm})^{1)}$ & 9.5 & 1.9 & $0.6-1.6$ \\
Field of view (mrad x mrad) & 64 (slit) x 64 (scan) & 64 (slit) 64 (scan) & $0.44 \times 1.34$ \\
Image size, full FOV high resolution (pix.) & $256 \times 256$ & $256 \times 256$ & - \\
Noise equivalent spectral radiance & $5.0 \times 10^{-4}$ & $2.5 \times 10^{-2}$ & $5.0 \times 10^{-4}$ \\
(central band, $\left.\mathrm{W} \mathrm{m}^{-2} \mathrm{sr}^{-1} \mu \mathrm{m}^{-1}\right)$ & Offner Relay & Offner Relay & Echelle \\
Spectrometer & $\mathrm{MCT}(\mathrm{HgCdTe})$ & $\mathrm{CCD}$ & $\mathrm{MCT}(\mathrm{HgCdTe})$ \\
Detectors &
\end{tabular}

${ }^{1)}$ depends on selected mode of operation; the finer value is shown here.
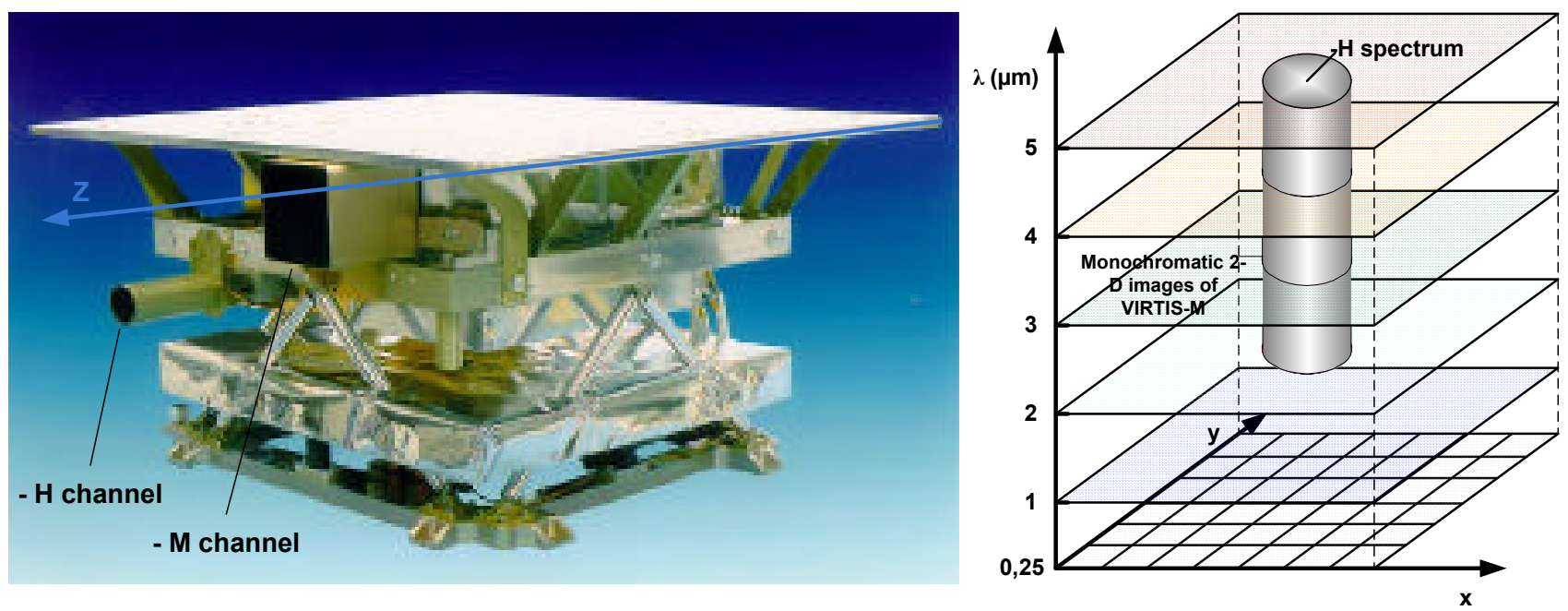

Figure 2. VIRTIS-ROSETTA. Left: Optics Module, credits: Officine Galileo, Italy, 2001; Right: VIRTIS imaging and spectral image cube recording ${ }^{70}$.

The output from VIRTIS-M is an image cube combining 2-D images with a spectrum from $0.28-5.13 \mu \mathrm{m}$ as shown in Figure 2 (right). The VIRTIS-H field of view is approximately centered in the middle of the -M image and provides spectra at high spectral resolution in this small portion of the image (Figure 2, right). The spectral sampling of VIRTIS- 
$\mathrm{M}$ is about $2 \mathrm{~nm}$ from 0.28 to $1 \mu \mathrm{m}$ and $10 \mathrm{~nm}$ from 1 to $5 \mu \mathrm{m}$, while for VIRTIS-H it varies between 0.6 and $1.6 \mathrm{~nm}$ (Table 1).

VIRTIS-M is characterized by a single optical head consisting of a Shafer telescope combined with an Offner imaging spectrometer and by two focal plane arrays (FPA). VIRTIS-H is a high spectral resolution infrared cross-dispersed spectrometer using prism and grating for dispersion. VIRTIS-H spectral resolving power is $>1000$. VIRTIS consists of four modules: the Optics Module containing the M- and -H Optical Heads, the two Proximity Electronics Modules (PEM) and the Main Electronics (ME). The Optics Module (Figure 2, left) is externally mounted on the -X panel of the spacecraft with the Optical Heads co-aligned in $+\mathrm{Z}$ direction. The slits of both optical systems are parallel to the $\mathrm{Y}$ axis. Each VIRTSI-M and -H channel has a cover to protect the instrument from direct solar illumination and to preserve the cold environment inside the spectrometer. The focal planes with state of the art CCD and infrared detectors achieve high sensitivity for low emissivity sources. VIRTIS-M-VIS uses a Si CCD (Thomson TH7896) for the range between 0.28$1.1 \mu \mathrm{m}$. The IR FPA of -M and -H are housed on bi-dimensional HgCdTe arrays of $270 \mathrm{x} 438$-pixel detectors designed to provide high sensitivity and low dark current ( $1 \mathrm{fA}$ at $80 \mathrm{~K}$ ). Therefore they are cooled to $70 \mathrm{~K}$ by an active cooler. VIRTIS-M and -H spectrometers themselves are cooled down to $135 \mathrm{~K}$ by means of a radiator reducing the background level of thermal radiation. ${ }^{36,40,70}$ The ROSETTA VIRTIS instrument is currently on its hibernation phase during its track to the comet 67P. ROSETTA will rendezvous with this small object in March 2014 in a distance of about 4 AU from the sun. ${ }^{74}$ VIRTIS-ROSETTA already produced high quality data during the Earth and Mars maneuvers (Earth: March 2005, November 2007, November 2009; Mars: February 2007) and observed the asteroids 2867 Šteins $^{58,74}$ and 21 Lutetia $^{61,91}$ flying through the main asteroid belt ${ }^{37}$.

Originally designed for the ROSETTA mission, VIRTIS was successfully applied in other space missions: the ESA cornerstone mission VENUS Express (VEX) and the NASA DAWN mission. Unlike the study of poorly differentiated objects like the targets of ROSETTA and DAWN, the ESA Venus Express mission is focusing on a highly differentiated terrestrial planet to answer general questions about the composition, structure, circulation, temperature, and dynamics of the Venusian atmosphere and surface. ${ }^{40,70}$ The VIRTIS-VEX instrument headed by Giuseppe Piccioni (Italy) and Pierre Drossart (France) is basing on the VIRTIS-ROSETTA development with some modifications. For the first time, VIRTIS-VEX investigates deep atmosphere and surface features of Venus systematically in the narrow nightside atmospheric windows from the orbit of the planet allowing to extract surface temperature and emissivity data on global scales. ${ }^{10}$ Features like the hot climate driven by a runaway greenhouse effect, the dense $\mathrm{CO}_{2}$ atmosphere, and the atmospheric superrotation distinguish Venus from the other terrestrial planets. Although the planet's mass and density differ only slightly compared to the Earth, both planets took completely different evolution paths. The VIRTIS-VEX data contribute to a better understanding of key processes in the evolution and formation of Venus by results, which are described exemplarily in Section 3.2.

\section{MErcury Radiometer and Thermal Infrared Spectrometer (MERTIS)}

Unlike Venus, the innermost planet Mercury, which was formed close to the sun, has a small mass of only 0.055 Earth masses. It did not develop an atmosphere comparable to other terrestrial planets, but it shows an unusual mean density. The ongoing NASA mission MESSENGER in Mercury's orbit started spectral measurements in the UV/VIS/NIR range. Preliminary photogelogic and spectral studies of MESSENGER's optical system show a heterogeneous surface. The VIS/NIR spectra demonstrate that Mercury is spectrally diverse and has a surface consisting of volcanically emplaced plains of various ages ${ }^{38,88}$ and terrains composed of older, less resurfaced material ${ }^{95}$.

In contrast to MESSENGER, the MErcury Radiometer and Thermal Infrared Spectrometer (MERTIS) onboard of the ESA mission BepiColombo going to be launched in 2014 will use the mid-infrared range for systematic compositional surface analysis from the orbit of the planet. ${ }^{11,53}$ MERTIS is a state of the art spectro-radiometer using an integrated instrument approach. It combines a pushbroom 7-14 $\mu \mathrm{m}$ IR grating spectrometer (TIS) with a 7-40 $\mu \mathrm{m}$ radiometer (TIR). TIS and TIR share the same optics, electronics, power, and in-flight calibration units. ${ }^{9,11}$ This approach is realized by a modular concept of the sensor head, electronic units, and power/calibration systems within a mass budget of $3.3 \mathrm{~kg}$ and power consumption less than $13 \mathrm{~W}$ nominal and $19 \mathrm{~W}$ under cold environmental conditions. ${ }^{9}$ The TIS is an imaging spectrometer with an uncooled micro-bolometer array. It has $160 \times 120$ pixel with a size of $35 \mu \mathrm{m}$ each. The optical system combines a three mirror anastigmat (TMA) with a modified Offner grating spectrometer. The grating is placed 
about midway between the slit and a concave mirror. The TMA integrates three off-axis mirrors with a second one as an aperture stop, and the Offner spectrometer consists of two concentrically spherical elements with a smaller one being the grating and a larger one being a large concave mirror opposed the grating. ${ }^{6,8,9,11,53,93}$ The optical design of MERTIS includes a telescope with a focal length of $50 \mathrm{~mm}$ and an F-number of 2 . The FOV will be $4^{\circ}$. The all over optical efficiency is $0.54 .^{8}$ The FOV will be imaged on the detector array resulting in an image cube where a line of the detector corresponds to the spectrum and a column to the two-dimensional spatial information of the target area scanned by the pushbroom device.

Table 2. Instrumental parameters of MERTIS. ${ }^{6,8,9,11,53,93}$

\begin{tabular}{lcc}
\hline & MERTIS-TIS & MERTIS-TIR \\
\hline Spectral range $(\mu \mathrm{m})$ & $7-14$ & $7-40$ \\
Spectral resolution $\lambda \Delta \lambda$ & $78-156$ & - \\
Spectral channel width & $90 \mathrm{~nm} /$ pixel & Line array $1: 7-14 \mu \mathrm{m}$ \\
& $4^{\circ}$ & Line array $2: 7-40 \mu \mathrm{m}$ \\
Field of view & $280-1400 \mathrm{~m}$ & $4^{\circ}$ \\
Ground sample distance $(400 \mathrm{~km})$ & 28 & $2000 \mathrm{~m}$ \\
Swath width $(\mathrm{km})$ & Bolometer matrix array & 28 \\
Detectors & $160 \times 120$ at $35 \mu \mathrm{m}$ & Thermophile line array \\
Number of pixel/size & $(100$ spatial, $80 \mathrm{spectral})$ & $2 \times 15$ at $250 \mu \mathrm{m}$ \\
Detectivity & NEP $<15 \mathrm{pW}$ & $\mathrm{D}^{*}=7 \times 10^{8} \mathrm{~cm} \mathrm{~Hz}^{1 / 2} \mathrm{~W}^{-1}$ \\
\hline
\end{tabular}

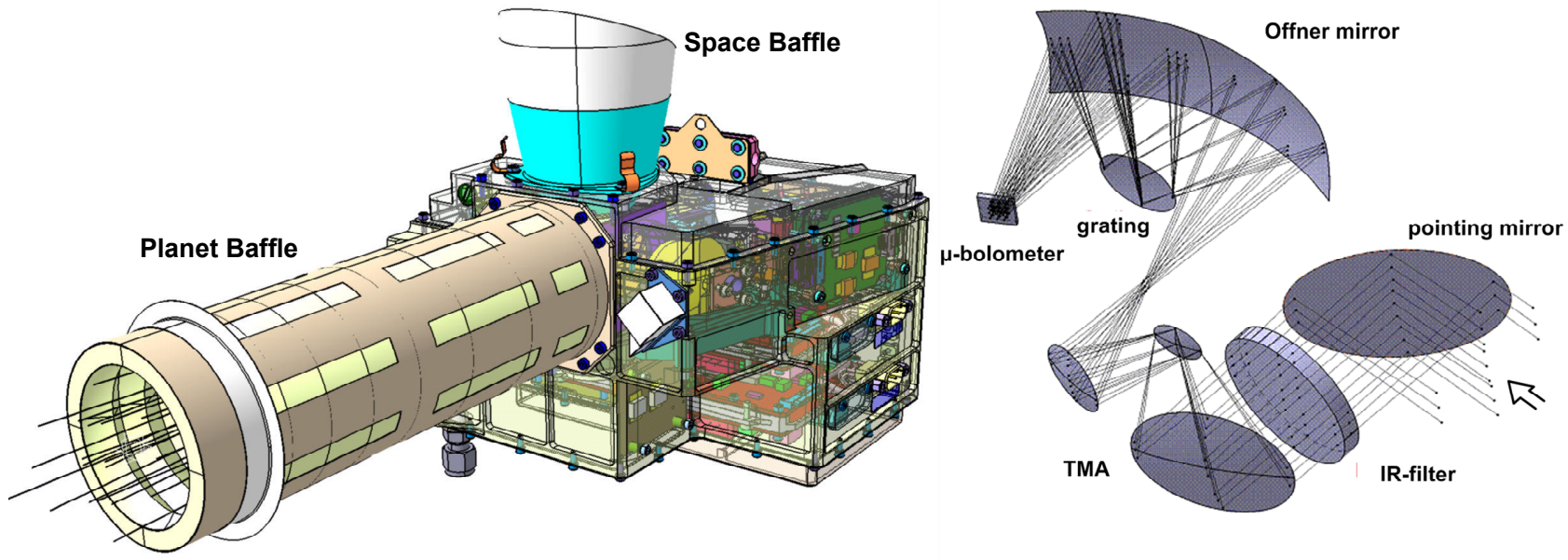

Figure 3. Left: MERTIS overall instrument design. ${ }^{11,93}$

Right: Optical scheme of MERTIS including TMA, pointing mirror, Offner spectrometer and detectors. ${ }^{8}$

Figure 3 shows the instrument overall design (left) and the optical scheme of MERTIS including TMA, pointing mirror, Offner spectrometer, and detectors (right). Table 2 summarizes main performance parameters of MERTIS. The TIS spectral band width will be $90 \mathrm{~nm} / \mathrm{pixel}$, and its spectral resolution will be in the range between $78-156$. This enables to resolve main diagnostic absorption bands in the emissivity spectra of Mercury's surface minerals. ${ }^{9,11}$ The spatial resolution for global mapping will be $500 \mathrm{~m}$ in the nominal case and better than that for $\leq 10 \%$ of the surface. At pericenter orbital altitude, the ground sample distance is between 280 and $1400 \mathrm{~m}$ with a swath width of $28 \mathrm{~km}$. For temperature monitoring, micro-radiometer thermopile arrays are placed on the sides of the entrance slit. Using the same 
optics, the pushbroom radiometer TIR is implemented operating in a temperature range between 150 and $700 \mathrm{~K}$ with a temperature resolution of $1 \mathrm{~K}$. This range covers the high dayside temperatures as well as the nightside temperatures where the planet surface is orientated to the cold space.

The onboard calibration of the instrument is realized by a pointing device, an internal black body (BB) calibration unit, and two instrumental baffles. The thermally shielded pointing device is located in front of the optical head. A rotary mechanism with a $45^{\circ}$ tilted mirror allows pointing to the internal BB at 300 and $700 \mathrm{~K}$, to a Space Baffle (SB), and to the Planetary Baffle (PB). The SB is orientated to the cold space whereas PB views the planet. The PB structure is designed to minimize straylight. ${ }^{98}$ Measurements of the $\mathrm{S} / \mathrm{N}$ with the Development Module of MERTIS revealed satisfying results for the radiometric resolution of the expected spectral features. ${ }^{11,93}$ Detailed descriptions of the whole instrument ${ }^{93}$, the optical elements ${ }^{12}$, and the $\mathrm{SNR}^{72}$ are given in accompanying papers (this issue).

\subsection{Planetary Fourier Transform spectrometers}

\section{Planetary Fourier Spectrometer (PFS)}

Due to the relative large halfwidths of characteristic absorption bands in the VIS/IR spectra of the solid surface, composition analyses of airless planetary surfaces require only moderate spectral resolution of $\sim 200$. In contrast to broad absorption features caused by electronic processes or lattice vibrations in the VIS to IR spectra of rocks, minerals or ices, the narrow bands of molecular vibrations in planetary atmospheres have to be resolved at much higher spectral resolution (> 1000). For studies of terrestrial planetary atmospheres, imaging spectrometers often provide limited information. Apart from the main goals of atmospheric research at high spectral resolution, the presence of materials in different aggregate states on planetary surfaces and in the atmosphere of the objects involves strategies to distinguish between them. A typical example is the cosmo-chemically important water in the solar system. From Mars it is known that water exists as solid material on the surface in the polar regions, or as subsurface permafrost as well as water vapor in the Martian atmosphere. One approach to separate the remote sensed signals of $\mathrm{H}_{2} \mathrm{O}$ in both aggregates is to measure at high spectral resolution.

Fourier Transform Spectroscopy is a valuable tool for spaceborne high resolution spectroscopy. The Planetary Fourier Spectrometer (PFS) for ESA's Mars Express mission is an infrared spectrometer optimized for atmospheric studies. ${ }^{42,43}$ PFS was developed by a consortium of several groups from Italy, Russia, Poland, France, Spain, and Germany. It is headed by the INAF-IFSI (Istituto di Fisica dello Interplanetario, Italy). The spectrometer is a double pendulum interferometer working in two wavelength ranges: the short wavelength channel (SW) from 1.25-5.5 $\mu \mathrm{m}$ and a long wavelength channel (LW) from 5.5-45 $\mu \mathrm{m}$. The interferometer uses retroreflectors (cube corners) generating the optical path difference by angular movement. The pendulum interferometer with full aperture is ideally suited for high throughput, adequate spectral resolution, and maximum compactness and robustness. ${ }^{4,52,43,54-56} \mathrm{~A}$ dichroic mirror reflecting all wavelengths lower than $5.5 \mu \mathrm{m}$ and transmitting higher wavelengths separates the incoming Martian radiation into two parts, which are afterwards directed to the corresponding interferometer channels. A band stop for lower wavelengths than $1.2 \mu \mathrm{m}$ is integrated in the SW. Each channel is equipped with a pair of cube corner mirrors attached by brackets to an axle angularly moved by a torque motor. Both channels use the same axle and drive mechanism as shown in Figure 4. The pendulum motion is controlled by a laser diode. The same laser diode generates the sampling signal for the A/D converter to measure the optical path differences of $608.4 \mathrm{~nm} .{ }^{43}$ Table 3 provides the main parameters of PFS. In front of the spectrometer, a two pointing axis device (scanner) allows the FOV to be pointed along or laterally to the surface and also to the internal black body for calibration. PFS is a modular instrument combining Optics Module, Electronics Module, black body system, scanner unit, and power supply. The SW uses a $\mathrm{CaF}_{2}$ beam splitter and a PbSe photoconductor detector. The LW integrates a CsI beamsplitter and a pyroelectric $\mathrm{LiTaO}_{3}$ detector. The interferograms recorded by PFS are double sided. ${ }^{42}$ The performance of the instrument results in a spectral resolution of $1.3 \mathrm{~cm}^{-1}$ in the entire spectral range. The spatial resolution at pericenter is up to $7 \mathrm{~km}(12 \mathrm{~km})$ for SW (LW) allows obtaining data about surface materials and temperatures only at quite coarse spatial scales. PFS data recording is still ongoing. There is an enormous amount of data available. One example discussed in Section 3.3. 
Table 3. Instrumental parameters of PFS. ${ }^{4,5,42,43,54-56}$

\begin{tabular}{lcc}
\hline & $\boldsymbol{S} \boldsymbol{W}$ & $\boldsymbol{L} \boldsymbol{W}$ \\
\hline Spectral range $(\mu \mathrm{m})$ & $1.2-5.5^{1)}$ & $5.5-45^{1)}$ \\
Spectral range $\left(\mathrm{cm}^{-1}\right)$ & $1700-8200^{1)}$ & $250-1700^{1)}$ \\
Spectral resolution $\left(\mathrm{cm}^{-1}\right)$ & $1.3^{1)}$ & $1.3^{1)}$ \\
Field of view $(\mathrm{deg})$ & $1.6^{1)}$ & $\mathrm{CsI}$ \\
Beamsplitter & $\mathrm{CaF}_{2}$ & \pm 5 \\
Max. opt. path difference $(\mathrm{mm})$ & \pm 5 & $\mathrm{CsI}$ \\
SW/LW separation & \multicolumn{2}{c}{ KRS-5 with multiple layered coating } \\
Interferogram & \multicolumn{2}{|c}{ double sided } \\
Spectral points & 8192 & 2048 \\
Detectors & photoconductor, PbSe & pyroelectric, $\mathrm{LiTaO}_{3}$ \\
Detector temperature $(\mathrm{K})$ & $210^{1)}$ & 290 \\
NEB $\left(\mathrm{W} \mathrm{cm}^{-2} \mathrm{sr}^{-1}\right)$ & $5 \times 10^{-9}$ & $4 \times 10^{-8}$ \\
\hline
\end{tabular}

1) corresponding to [43]
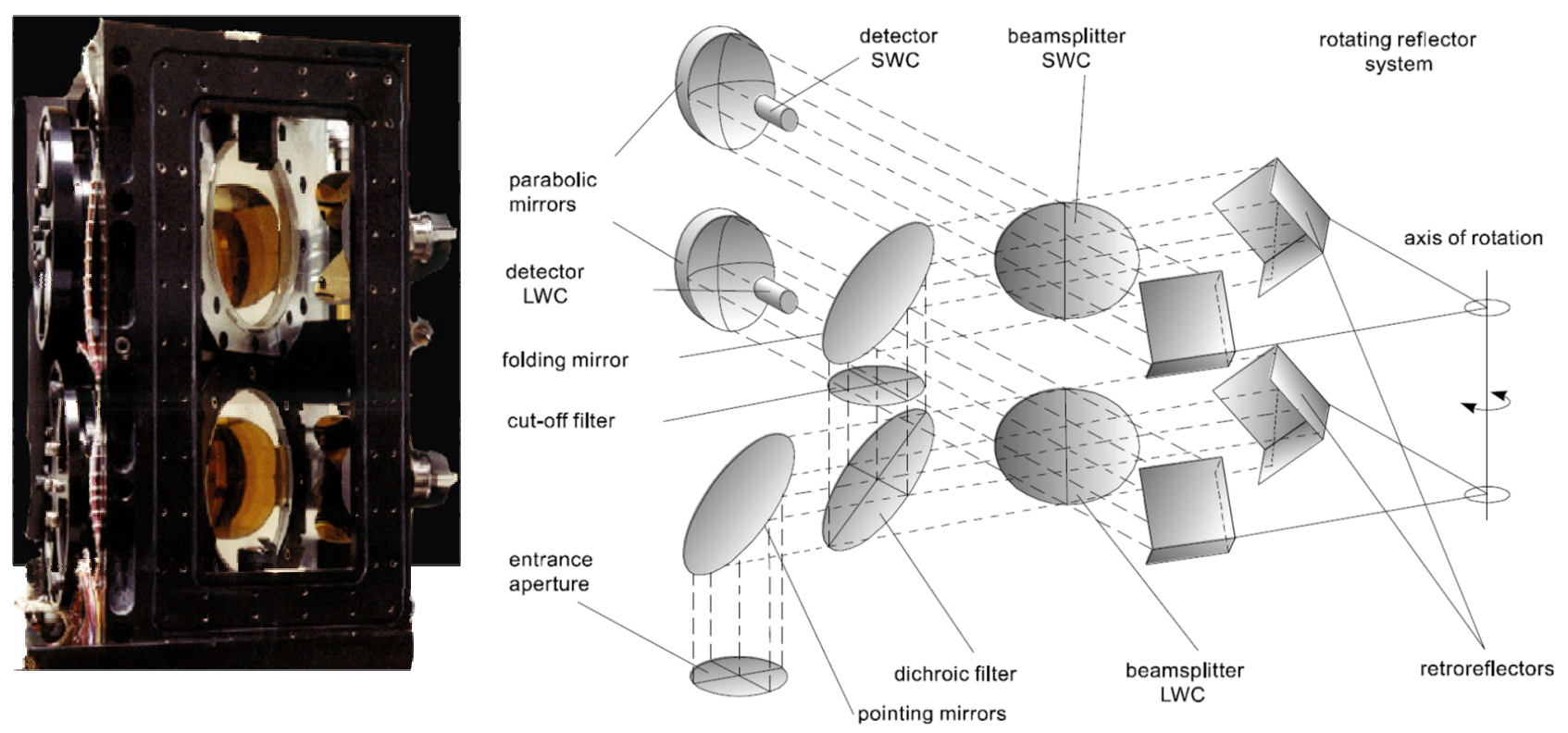

Figure 4. Left: PFS Engineering Model; Right: Optical design of $\mathrm{PFS}^{56}$.

\section{SPECTRAL STUDIES OF PLANETARY SURFACES}

The rocky bodies in the solar system display a great variety of surface and crustal composition. The surfaces of the terrestrial planets, moons, and small bodies are composed of rocks and/or ice. As seen in Figure 1, large variations in albedo and emissivity exist that are mostly related to compositional variations. The different bodies are characterized by unique geologic features giving hints to surface forming processes like meteoritic bombardment, volcanism, tectonics, and weathering processes. The cross correlation of geologic and compositional units cast light on the origin and evolution of the different objects. The terrestrial planets and many moons and asteroids have undergone differentiation. 
The composition of a differentiated body and the location of its formation are important factors controlling the evolution and the nature of its crust. The enrichment of $\mathrm{Mg}$ or Fe in the body's mantle decides about the silica content of magma and lava flows. Finally, volatiles in the mantle support the formation of an anorthositic crust. ${ }^{25}$ Comparative planetology contributes to study processes of planet formation including Earth.

Spectral remote sensing is a powerful tool for the geochemical analysis of planetary surfaces. In most cases, the remote study is the only possibility to extract compositional data. During the last decades, all terrestrial planets, some moons, and minor bodies have been targets of different space missions offering the possibility to study them close to the objects. The reconnaissance and global surveys from orbit of the body or during a fly-by currently form the basis for compositional analysis on global scales. The most common applied technique is the passive remote sensing. This method was complemented by active measurement methods (laser and radar techniques), Gamma spectroscopy, and geochemical analyses at the surface of some of the objects. The elemental and mineralogical in-situ studies onboard landing systems (Mars, Venus, comet Wild) provided an important ground truth for the calibration of spectral remote sensing data.

The instruments that have been introduced in the previous section were contributing to this effort. Designed to investigate different targets, they facilitated numerous scientific studies of planetary surface. The following subsection gives only a small selection of prominent results that stress the importance of this method in comparative planetology.

\subsection{Small bodies}

Minor bodies like asteroids and comets have preserved information about the primary material forming our solar system. They are poorly differentiated and indicative of the composition of different environments of the primordial solar system. Minor bodies represent material that has not contributed to the formation of highly differentiated objects, but left over from the early accretion phases of the proto-planetary disc. In this regard they contain records of the solar nebular conditions.
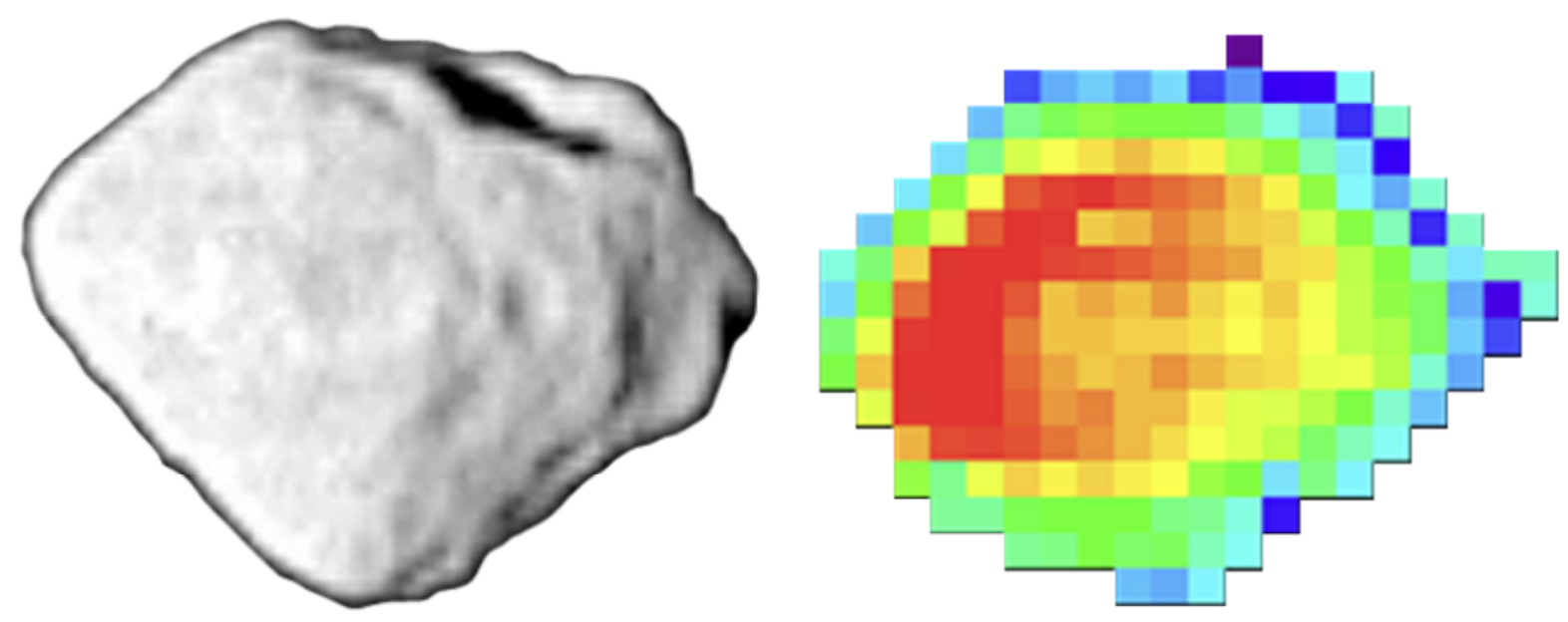

Figure 5. Šteins during the Rosetta encounter 2008.

Left: OSIRIS image, Credits: ESA MPS for OSIRIS Team MPS/UPM/LAM/IAA/RSSD/INTA/UPM/DASP/IDA. Right: VIRTIS thermal map, maximum temperature $230 \mathrm{~K}$ (red), Credits: ESA, IFSI, VIRTIS Team ${ }^{36}$.

The ESA's cornerstone mission Rosetta has the objective to study small bodies in the solar system. On its way to the comet 67P/Churyumov-Gerasimenko, the Rosetta mission passed the asteroid main belt between 2.2 and 3.4 Astronomical Units (AU). In September 2008 Rosetta encountered the asteroid 2867 Šteins (Figure 5) at 2.41 AU and at a distance of $800 \mathrm{~km}$. Rosetta's relative speed with respect to Šteins was $8.6 \mathrm{~km} / \mathrm{sec}$. Šteins is one of the 24 known rare E-type asteroids. Their mineralogy is dominated by the silicate entstatite $\left(\mathrm{Mg} \mathrm{SiO}_{3}\right) \cdot{ }^{33}$ E-type asteroids have high albedos with a mean of 0.44 . Their spectra are generally flat with weak absorptions and lack of FeO-bands. Among the E-type 
asteroids, which are classified into four subgroups, Šteins belongs to the E[II] subgroup. ${ }^{44}$ The spectrum of an E[II] asteroid is characterized by spectral absorption bands near 0.49 and $0.96 \mu \mathrm{m}$ and a flat spectral continuum above $0.6 \mu \mathrm{m}$.

The VIRTIS spectrometer onboard Rosetta collected spectrally, spatially, and temporally resolved observations of the asteroid and performed spectroscopic measurements in the range between 0.25 and $5 \mu \mathrm{m}$ during the fly-by. The spatial resolution on the asteroid surface was in the order of $200 \mathrm{~m} /$ pixel for VIRTIS-M. ${ }^{36}$ The close-up detection of VIRTIS-M revealed spatially resolved spectra of the asteroid and data above $2.5 \mu \mathrm{m}$ that are usually not available from Earth based observations. The measurements of VIRTIS show that Šteins is spectrally homogeneous. The appearance of bands at $0.49 \mu \mathrm{m}$ and $0.96 \mu \mathrm{m}$ could be confirmed. They may arise either from sulfide minerals found in aubrites and enstatite chondrites or Ti-bearing components for the 0.49 feature. ${ }^{26,75}$ Between 1 and $4 \mu \mathrm{m}$, the VIRTIS spectra are almost flat. Additional bands between 4 and $5 \mu \mathrm{m}$ have been evidenced for the first time. The nature of these bands is still unknown. At wavelengths above $4 \mu \mathrm{m}$, the thermal emission starts to exceed the reflected sunlight. This range can be used to extract information about the surface temperature of the asteroid, which was determined to lie between 200 and $230 \mathrm{~K}$ (Figure 5, right). ${ }^{36} \mathrm{E}[\mathrm{II}]$-asteroids may be composed of basalts from E-chondrite-like parent bodies, which underwent partial melting. ${ }^{62}$ The new data will improve our knowledge about the link between E-type asteroids as parent bodies for aubrites/ entstatite achondrites and the formation of these objects. The high optical albedo at zero phase angle is a sign of a regolith covered surface.

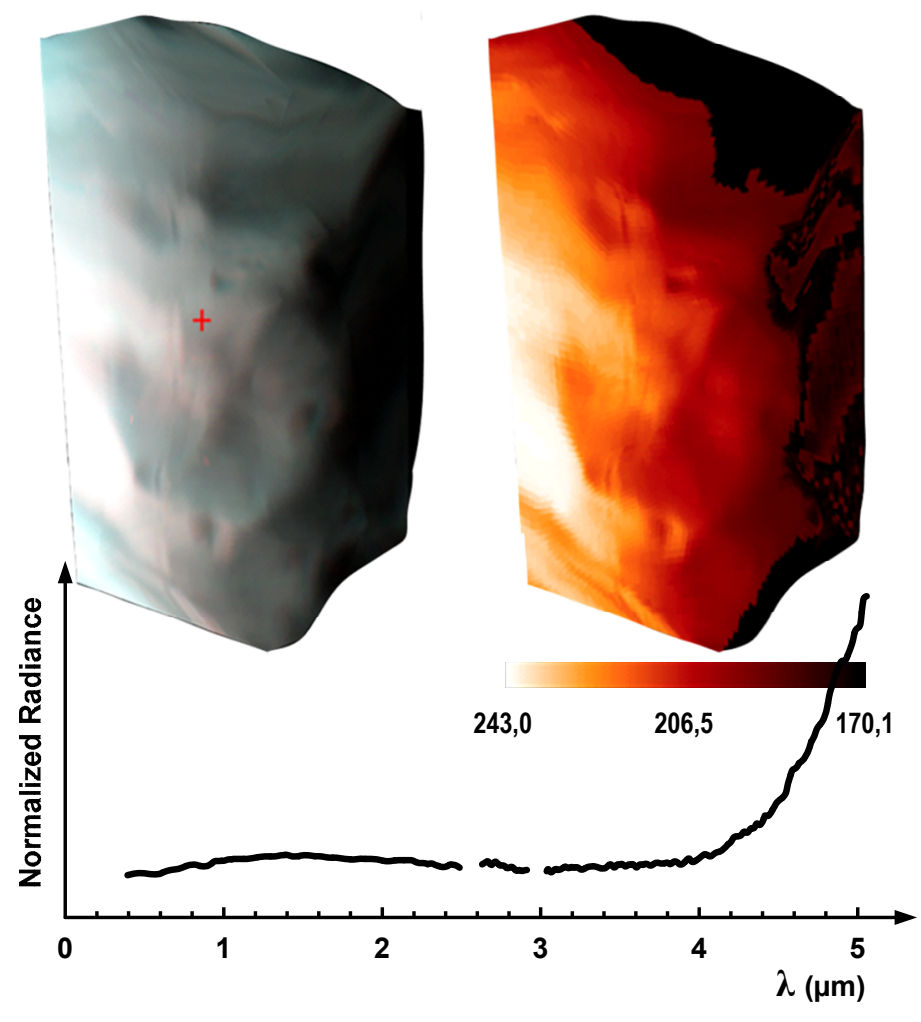

In July 2010 Rosetta encountered the asteroid 21 Lutetia (2.436 AU semi-major axis) with a closest approach at a distance of $3162 \mathrm{~km}$. Rosetta passed the asteroid at $15 \mathrm{~km} / \mathrm{sec}$. During the fly-by, VIRTIS obtained hyperspectral images, spectral maps, and temperature maps of the asteroid. ${ }^{37}$ Figure 6 shows a false color map of Lutetia composed from three VIRTIS-M-IR bands taken in the pushbroom mode (Figure 6, left top side). Thermal emission sets in above $4 \mu \mathrm{m}$. A temperature map was created according to a thermophysical model. ${ }^{39}$ It is shown on the right top side of Figure 6. Below the maps, a mean disk-integrated VIRTIS-M spectrum is presented. $^{37}$ The surface of Lutetia is dominated by lunar-like regolith similar to Šteins. The spectra taken by VIRTIS do neither display hydration bands, nor absorptions characteristic for Fe-bearing minerals. Even though many questions are still unanswered, 21 Lutetia seems to be an unaltered remnant of the primordial planetesimal population composed of chondritic material, of enstatite, or of carbonaceous species. ${ }^{37}$

Figure 6. Lutetia during the Rosetta encounter 2010.

Left top: VIRTIS-M, false color image (B-2 $\mu \mathrm{m}, \mathrm{G}-4 \mu \mathrm{m}, \mathrm{R}-5 \mu \mathrm{m})$, the cross marks the North Pole location;

Right top: VIRTIS temperature map ${ }^{37}$; Bottom: Mean disk integrated VIRTIS-M spectrum ${ }^{37}$; Credits: ESA, INAF, VIRTIS Team, [Coradini et al., ${ }^{37}$ ].

\subsection{Terrestrial surfaces - Venus}

Unlike the primordial minor objects, terrestrial planets are highly differentiated objects. They were formed in a series of dynamical steps in the protoplanetary disk around the sun 4.5 billion years ago. Starting from micron-sized dust grains, $\mathrm{km}$-sized planetesimals were formed by concentration of material and collision grow. Once solid bodies reached the 1 
$\mathrm{km}$ size, gravitational interactions became significant creating bigger objects. The terrestrial planets formed in the inner solar system depleted by volatiles, and therefore they are composed primarily of refractory material: silicate rocks and/or metals. As a result of a three step evolution including random velocities and gravitational focusing, runaway growth, and oligarchic growth, the planetary embryos accreted. It followed a late state of heavy meteoritic bombardment finalizing the mass growth. ${ }^{30,71}$ Once this process ended, the planet differentiated, forming the core, the mantle, and the crust. The primary crust was then rebuilt as a result of meteoritic impacts, volcanism, tectonics and weathering processes. Planetary atmospheres originated during this period of evolution. Despite the common basis of formation processes, the terrestrial planets display a large variability in terms of their actual state and the evolutionary paths.

Venus is the second planet from the sun, formed close together with the Earth. Both planets are similar in size, gravity, and bulk composition. However Earth and Venus followed completely different evolutionary paths. Venus has an extremely dense atmosphere mainly consisting of carbon dioxide causing surface temperatures up to $460^{\circ} \mathrm{C}$ and surface pressures about 92 times higher compared to Earth. A thick cloud layer of sulfuric acid droplets generates a strong greenhouse effect and reflects the major part of incident solar radiation at the Venusian dayside. ${ }^{90}$ The atmosphere of Venus is opaque in most wavelength ranges. For this reason, the surface of Venus is poorly studied. The Magellan spacecraft measured the topography of Venus for the first time using radar waves penetrating the dense atmosphere with a SAR system. ${ }^{41}$ The Magellan radar data indicate a global resurfacing by a violent volcanism in the younger history of the planet about $500-700$ million years ago. ${ }^{14-17,64}$ The volcanic landforms are consistent with low-viscosity eruptions, which are characteristic of mafic rocks like basalt. ${ }^{48}$ The discovery of possible recent volcanism demonstrates that residual volcanic processes may remain active until the recent past. ${ }^{76}$ However, the Magellan data and chemical studies of landers also suggest that some high-viscosity lava formed pancake domes and festoons ${ }^{63,90}$, which could be related to the presence of more felsic materials like rhyolite. The slightly lowered microwave reflectivity measured by Magellan at moderately high elevations, specifically for so-called tesserae, was discussed in connection with felsic materials which display lower values of reflectivity compared to mafic components. ${ }^{59}$ This was supporting geophysical hypotheses about a felsic crust. ${ }^{69}$ Estimates have demonstrated that there may be significant spatial variations in the surface emissivity as large as $20 \%$, which would correspond to the difference between granitic and basaltic rocks. ${ }^{46}$ Identification of residual felsic tesserae components would give a chance to study the pre-resurfacing history of the planet. ${ }^{10}$ As far as the radar signal is not very sensitive to the physical and chemical properties of the surface, its composition is unknown on global scales, and the majority of our knowledge about the surface material was obtained by the few landers of the early Venera and Vega missions.

The discovery of the nightside near-infrared atmospheric windows between 0.8 and $2.5 \mu \mathrm{m}^{1,2}$ provided a new technique for studying the lower atmosphere of Venus and its surface. VIRTIS is the first imaging spectrometer in the orbit of Venus systematically addressing this issue. VIRTIS contributes to these studies using VIRTIS-M-IR for mapping the surface temperature and estimate the surface emissivity by observations in the 1.0-1.2 $\mu \mathrm{m}$ spectral windows. Figure 7 (top) illustrates that around $96 \%$ of the measured nightside emission in the window $1(1.02 \mu \mathrm{m})$ results from the surface. The radiance contributing from the surface in the windows 2 and $3(1.10$ and $1.18 \mu \mathrm{m})$ is $57 \%$ and $48 \%$, respectively. Observed surface temperature variations are almost exclusively due to topographic changes. ${ }^{10,47}$

Detailed quantitative data analyses and the retrieval of surface features from each individual spectrum require the use of extensive radiative transfer simulations tools. Most techniques have been developed so far for low and moderate temperature and pressure atmospheric environments of Earth, Mars, and Venus mesosphere above the cloud cover. They cannot be adopted without major modifications to model the extreme environmental conditions in the deep atmosphere of Venus. ${ }^{22,47,65,92}$ For the extraction of quantitative surface data, new approaches have been implemented to radiative transfer simulations. The latest updates also apply a refined Magellan ephemeris and a preprocessing of topography data to incorporate atmospheric blurring. The details of this approach are described in papers [67], [47], and [57].

VIRTIS-M-IR measurements provide strong indications for the heterogeneity of the surface composition of Venus. Surface emissivity variations may be due to local changes in chemical composition (mineralogy), texture, and grain size of rocks. Physical and chemical weathering on the surface of Venus is another important source of emissivity anomalies that would cause changes in the measured spectra. ${ }^{18,19,76}$ Emissivity retrievals using the $1.02 \mu \mathrm{m}$ window for a couple of orbits that exhibit strong topographical variations over the northern hemisphere suggest lower surface emissivity at higher elevation areas ${ }^{47}$ as it is illustrated in Figure 7 (bottom). Iron-rich mafic minerals tend to form pronounced $\mathrm{Fe}^{2+}$ absorptions at this wavelength. ${ }^{27}$ As far as felsic minerals are mostly spectrally neutral at $1 \mu \mathrm{m}$, they might display a 
lower emissivity (higher reflectivity) compared to the iron-rich mafic materials. In case tesserae highlands are more felsic, as proposed by Nikolayeva et al. ${ }^{69}$, this behavior may give a hint on residual older highland material. Müller et al. ${ }^{67}$ and Helbert et al. ${ }^{51}$ did not comment on such a general trend, but they also found emissivity values being generally lower at tessera terrains. Once the presence of an older felsic component is confirmed, this will allow gaining insights into the pre-resurfacing history of Venus' surface.
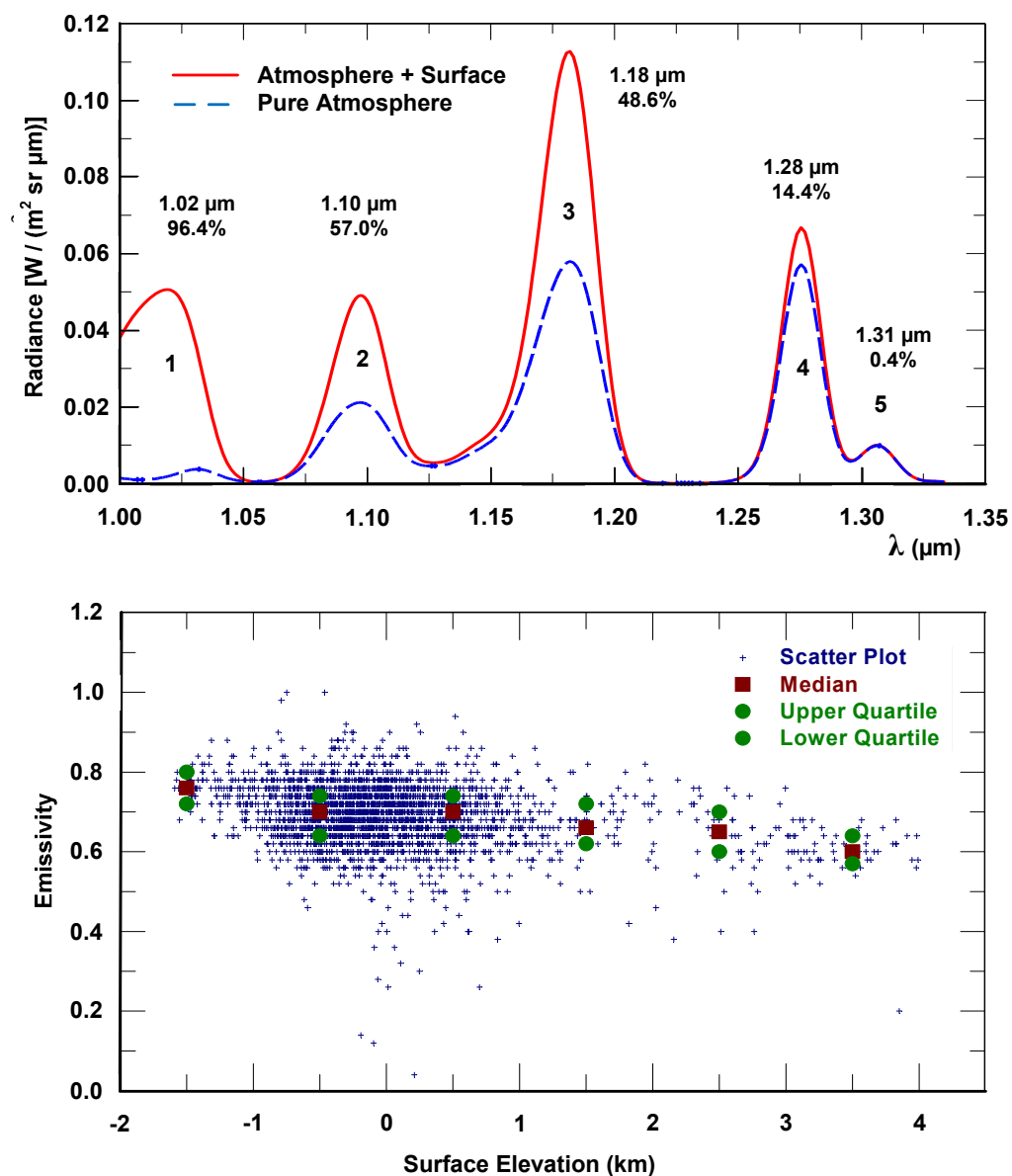

Figure 7. Top: Simulated surface radiance contribution to the total nightside emission in the atmospheric windows between 1.0 and $1.35 \mu \mathrm{m}$ that are observed by VIRTIS-M-IR ${ }^{47}$ Solid line: Atmospheric and surface radiance, broken line: Pure atmospheric radiance. Bottom: Retrieved surface emissivity from VIRTIS-M-IR data at $1.02 \mu \mathrm{m}$ for 47 orbits over the northern hemisphere as a function of surface elevation. ${ }^{47}$ Solid boxes and circles mark the medians, and upper and lower quartiles of $1 \mathrm{~km}$ surface elevation bins.

An approach started currently to apply the presented new algorithms of data evaluation and radiative transfer simulation to the VIRTIS-M-IR data obtained over the southern hemisphere of Venus. The southern hemisphere is observed during the apocenter passage of Venus Express providing imaging data. The use of larger areas that were partially repeatedly observed will improve the estimation of the different trends in surface temperature and emissivity and contribute to a clearer understanding of the discovered trends.

\subsection{Terrestrial surfaces - Mars}

Mars is the best explored planet among the terrestrial ones so far. Mars has a mass which is 0.107 of Earth's mass and a mean density of $3.93 \mathrm{~g} \mathrm{~cm}^{-3}$. Mars is today a desert and cold planet. Its surface pressure below 10 mbar does not allow liquid water to be present on its surface. Water presently exist either as solid material (e.g. at the poles) or as water vapor in the atmosphere of the planet. Our outer fellow planet has been a target object of many orbital space studies and 
different landing systems. With the ground data correlation and the knowledge coming from SNC meteorites, it succeeds to record the chronology by the SFD (crater Size Frequency Distribution) method. ${ }^{13}$ Martian history is divided into periods based on stratigraphic relations and geologic processes. ${ }^{89}$ The Noachian period with ages between 4.5 and 3.7 billion years is divided in an early phase (4.5-3.95 Ga ago), a middle phase (between 3.95-3.8 Ga ago), and a late phase (3.8-3.7 Ga ago). ${ }^{45}$ The Hesperian Period covers the time between 3.7-3.0 Ga ago, subdivided into an early (3.7-3.6 Ga ago) and a late phase (3.6-3.0 Ga ago). This period is followed by the Amazonian phase divided into the early (3.0-1.8 Ga ago), the middle (1.8-0.5 Ga ago), and the late (from 0.5 Ga ago) period. Different geologic features can be assigned to these periods like cratering, volcanism producing the highest shied volcanoes known in the planetary system, flow and canyon structures like the $4000 \mathrm{~km}$ long Valles Marineris, deposit layers, and polar caps.

The team operating the Omega spectrometer on ESA's Mars Express has extensively studied the surface of Mars within the different areas depending on their ages. ${ }^{23}$ Omega is a precursor for the VIRTIS development. It maps the Martian surface composition between 0.25 and $5 \mu \mathrm{m}$. Based on these studies, the surface mineralogy was used to classify the ages of surface areas. This is an independent and alternative model compared to cratering record. The Omega studies have shown that phyllosilicates mostly appear in the older southern hemisphere of Mars which is attributed to the Noachian period. Phyllosilicates are weathering products of the primary basalts indicating the presence of liquid water at the surface during their formation. The phyllosilican era corresponds to a time when the planet was warmer and the atmosphere was denser and wet. At the end of Noachian phase, episodic volcanic eruptions started to resurface major parts of the northern hemisphere. With the transition to the Hesperian phase, the mineralogy of the corresponding areas is dominated by materials containing sulfates.

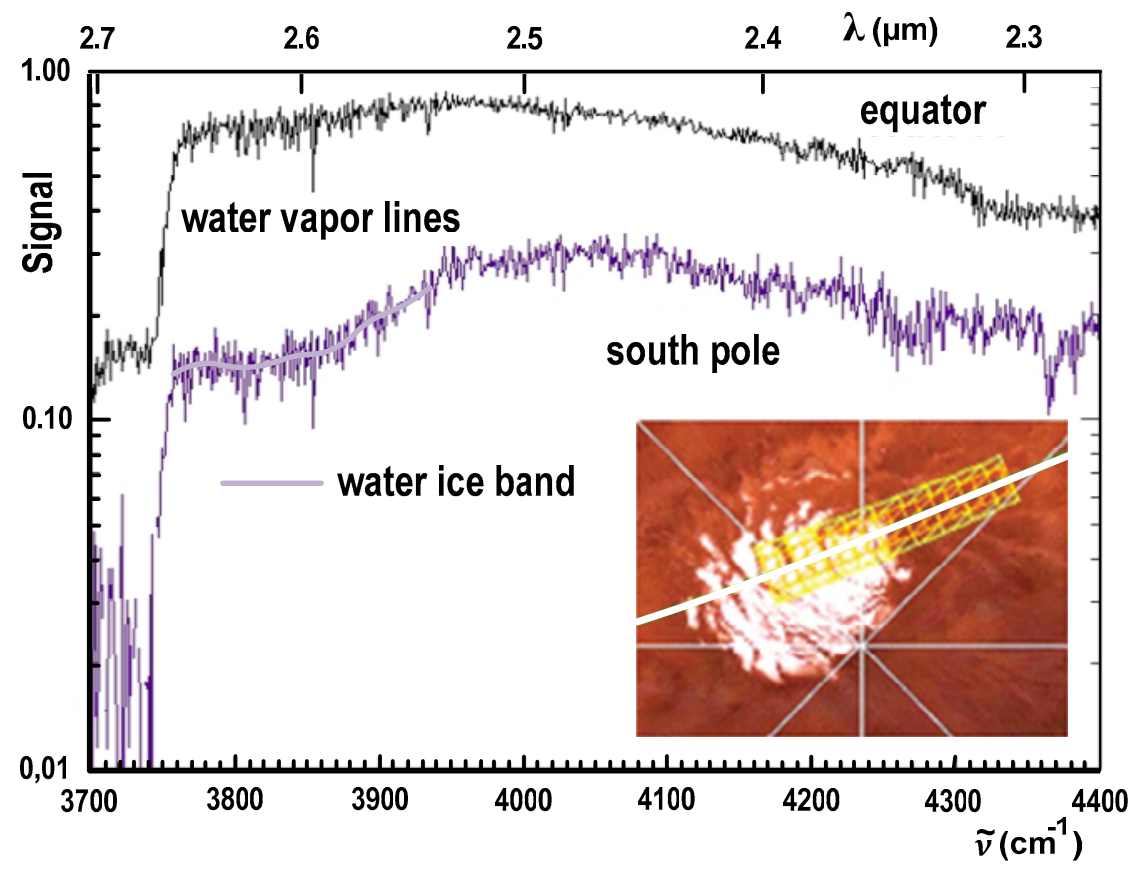

Figure 8. Measurements of PFS over the South Pole of Mars. Credits: ESA, Formisano and the PFS Team.

The sulfates are weathering products of the phyllosilicates indicating a degradation process without liquid water in a dry environment. This phase is named Theiikian era. The younger terrains on Mars are deposited with highly oxidized material which are anhydrous ferric oxides generating the red-brown color of the soil. Ferric oxides are the tertiary weathering products at the end of the degradation chain under dry conditions. The spectral studies of Omega have been supported by many other spectral studies like TES ${ }^{31}$, THEMIS ${ }^{32}$, and CRISM ${ }^{68}$. They have shown the capability of spectral remote sensing in reconstructing the evolution paths of planetary surfaces. 
It seems that the evolution of Mars is strongly linked to the history of the water. Signs of early liquid water can be found photogeologically or with spectral methods. The Omega spectral measurements suggest, that the warmer and dryer phase on Mars was a short period in Noachian. Obviously the planet lost its atmosphere very early, becoming dry and cold. The exploration of the today's water cycle gives important input to understand the processes of the early climate change on Mars. The Planetary Fourier Spectrometer contributed to these analyses by separating spectral information of water in the atmosphere and at the ground using measurements performed over the polar caps. Figure 8 demonstrates this principle by an example of a PFS observation. The water ice at the pole cap causes a broad absorption band in the PFS spectrum, whereas the water vapor is generating narrow lines of different continuum level.

\subsection{Terrestrial surfaces - Mercury}

Mercury is an unusual member of terrestrial planet family, and it holds an important place in understanding the formation of our inner planets. The innermost planet of our solar system is the smallest, at about $40 \%$ of Earths diameter and little more than $5 \%$ of its mass. Its relatively high mean density of $5.4 \mathrm{~g} \mathrm{~cm}^{-3}$ indicates a large metallic core of 60 $70 \%$ of the total mass. ${ }^{29,87}$ The existence of a weak magnetic dipole field can be a sign that the metallic core is still partially molten. The magnetic field of Mercury could be a remnant resulting from the cooling of the exterior part of the primary planet in the presence of an internal magnetic field, or it could be a residual dynamo. In spite of the observed magnetic field, which implies ongoing internal activity of this small planet, its surface is dominated by impact craters similar to the moon. Although volcanic vents affected the surface structure during and shortly after the Heavy Meteoritic Bombardment (HMB), the surface of Mercury is old. ${ }^{49,50}$ Additionally, the absence of a $1 \mu \mathrm{m}$ absorption band in the spectra of Mercury's surface indicates that it is depleted in FeO. ${ }^{94,95}$ Several hypotheses have been proposed to explain these unusual characteristics including a giant impact removing parts of the silicate crust from the planet, ${ }^{20,21,29,97}$ vaporization processes of the silicate and mantle, or an equilibrium condensation in the solar nebular ${ }^{28,96}$. These hypotheses lead to different predictions for the bulk chemistry of the planet. Comparative models have to account for the observed anomalies. A key information providing inputs to these models are studies of the surface composition of Mercury. Very little is known about it. Earth based observations of Mercury are complicated due to the small angular distance of the object from the sun.

Located close to the sun, the planet is also difficult to target by spacecrafts. After the first fly-by at Mercury with Mariner 10, the MESSENGER spacecraft is operating in 2011 in the orbit around Mercury. MESSENGER houses the Mercury Dual Imaging System (MDIS) and the Mercury Atmospheric and Surface Spectrometer (MASCS, 0.115-1.45 $\mu \mathrm{m}){ }^{24,38}$ The joint investigations of this complex will reveal global information about the surface geology and VIS/NIR spectral maps of the planet. These studies are the precursor for the ESA cornerstone mission BepiColombo to Mercury to be launched in 2014. Complementary to VIS/NIR instruments, BepiColombo will be equipped with the first planetary imaging MIR-spectrometer operating at Mercury. The MERTIS device that has been presented in Section 2.1 aims at four general science objectives: (a) study of Mercury's surface composition, (b) identification of rock-forming minerals, (c) global mapping of the surface mineralogy, and (d) study of surface temperature and thermal inertia. Already Earthbased observations have shown the capability of MIR-spectral remote sensing of Mercury. ${ }^{77-86}$ The mid-IR range offers a mineralogical identification, classification, and mapping by characteristic spectral features: Emissivity Maximum (EM, Christiansen frequency), Reststrahlen Bands (RB), and Transparency Features (TF) ${ }^{6,8,9,11,53,77-86}$ MERTIS measures the thermal emission of Mercury between 7 and $14 \mu \mathrm{m}$. It is known from Earth-based analyses that the mid-IR spectral characteristics are heterogeneous. The surface mineralogy seems to be dominated by Na-rich plagioclase, and Ca- and Mg-rich clinopyroxenes. Additionally, orthopyroxenes and dark components appear, the origins of which are uncertain.

The dayside surface temperature of the innermost planet rises up to $700 \mathrm{~K}$, while it cools down to $100 \mathrm{~K}$ at nightside. For comparison of the MERTIS data with spectra of terrestrial analogous and meteoritic materials, the creation of a new spectral catalog is in progress. It will contain emissivity spectra for relevant temperatures up to $700 \mathrm{~K}$ at the Planetary Emissivity Laboratory (PEL) ${ }^{52}$ A detailed description of these laboratory measurements, examples of relevant spectra, and resulting observation and identification strategies of rock-forming minerals are given in an accompanying paper ${ }^{11}$ in this issue. 


\section{PROSPECTS AND QUESTIONS FOR FUTURE OBSERVATIONS}

Spectral studies of solid planetary surfaces contribute to answer major questions regarding processes of the origin, evolution, and the actual state of the planets, moons, and minor bodies of the solar system. Comparative planetology integrates and cross-connects these data to explain the object, its geology, composition, climate, and physical state within a common framework. Many aspects directly affecting our knowledge about the state and evolution of the Earth were extracted on this base. Comparing the terrestrial planets, one of the mysteries is the divergent evolutionary path of each of the planetary objects. An important point of interest is the study of the current similarities and differences of the single bodies in all its facets. These data are the starting basis for modeling the processes of their origin. In this context, spectral remote sensing contributes to determine compositional, thermal, and physical data. The primordial small bodies in the solar system provide important information about the ancient, lowly differentiated material that was forming the terrestrial objects.

Most common theories of terrestrial planetary formation follow the progression discussed in the previous section, in which a cloud of gas and dust undergoes gravitational collapse to form a protoplanetary disk of material which aggregates to form planetesimals and later on, the planets. Basing on this assumption, the early terrestrial planets could have been more similar when they were formed than they are today. The second basic question resulting from this scenario is why they underwent dramatically different evolutionary paths? What are the main reasons for this? Differences in the initial mass and composition, location of formation, migration processes in the early planetary system, and catastrophic events may have contributed to this development. The geologic activity has left characteristic features at the surfaces of the terrestrial planets that are associated with material rising up from the interior of the planet. Stratigraphic and geochronologic techniques date the different surface areas. Spectral studies allow discussing environmental weathering conditions on primary planets up to the recent past within these areas.

The comparison of the terrestrial planets shows that the final surface forming processes on Mercury finished shorly after the HMB phase, while Mars was episodically active by volcanic vents until the younger past. Venus has been resurfaced about 500-700 billion years ago and may be locally active until today. The atmospheres of the terrestrial planets give insights into the climate histories and the evolutionary paths after formation of the primary planetary object. The small Mercury could not preserve an atmosphere in the vicinity of the sun. Mars obviously lost the major part of this atmosphere very early within the Noachian phase due to extensive escape of atmospheric constituents into the interplanetary space. The dense atmosphere of the slow retrograde rotating Venus, whose dry surface is permanently heated up by a runaway greenhouse effect, superrotates the clouds in only four days around the equator.

Some of the open questions in the formation of the solid objects in the solar system drive the performance of future missions. Collisions of planetesimals in the late HMB had a significant impact on the physical properties of planetary orbits and the resonances in the solar system. One of the mysteries is the retrograde and slow rotation of Venus that may be associated with such an impact. Moreover, different baseline conditions during the formation of the primary objects are reflected in different current states of the terrestrial planets. This requires more information about the interiors. A contribution to improve models of the interior and the magnetic field of terrestrial planets is the collection of valid data about the composition (mineralogy, elemental abundances) of the planetary crust. The study of the different geologic chronology and the nature of geophysical processes (tectonics, volcanism) is another important question that has to be answered by reconstructing the sequences of processes after the formation of the primary crust. Studies of planetary atmospheres, i.e. investigation of mechanisms controlling their mass, water content, interaction with the solar wind and interplanetary space, cloud chemistry and physics, atmospheric escape, and atmospheric response to volcanism will help to estimate the climate and environmental history of the different bodies. Finally, the history of water in the inner planetary system remains an open question. The early inner parts of solar system were originally depleted in volatiles. Minor objects could be subject to transport water into the inner regions of the planetary system. The formation of giant planets probably perturbed volatile planetesimals from the region of the planets beyond the ice boundary into the inner solar system, providing volatile material to be incorporated into the material forming the terrestrial planets. ${ }^{66}$

Spectral measurements during future space mission will be able to contribute to solve some of these issues. This includes systematic mapping of the surface mineralogy of solid planetary surfaces, linked to the geologic chronology, determination of surface ice properties, studies of dynamic phenomena (temporal, seasonal, and regional) as well as analyses of atmospheres (composition, structure, clouds, temperature profile, and dynamics). The investigation of water 
in the solar system and the study of its history are of particular importance. The discovery of a possible subsurface ocean on Jupiter's moon Europa, the detection of other icy worlds in the outer planetary system, ice deposits on permanently shadowed areas at the poles of Mercury and the moon suggested to explain the measured unusual radar signals, as well as the water cycles on Mars require more attention to studies of the water history in the solar system. All together, the future spectral planetary remote sensing will require a close networking of systematic mapping and spectrally high resolution observation strategies as well as appropriate instrumental solutions. For studies of the icy outer moons, a wavelength extension of spectral imaging spectrometers is required to fill the observation gap between 5 and $7 \mu \mathrm{m}$. This effort will enable to measure the H-O-H fundamental deformation vibration band of water near $6.25 \mu \mathrm{m}$ that allows studying different ice-water configurations. As far as future experiments on landing devices will collect in-situ information about elemental and solid state characteristics of materials at selected areas on different objects, references will be available to link and calibrate the remotely sensed spectral data.

\section{CONCLUSION}

The development and application of advanced UV/VIS/NIR/TIR imaging spectrometers like VIRTIS, MERTIS, and FTIR spectrometers like PFS for planetary exploration on deep space missions opened up the way for simultaneous observations of planetary surfaces at high spatial and high spectral resolution. Scientific questions in planetary physics and extreme conditions to operate the instruments in space environment have driven innovative technical and engineering solutions. Compact and integrated optical systems have been applied including Offner grating spectrometers for pushbroom imaging or cube corner pendulum optics for planetary interferometers. High sensitivity detector systems for the IR range have been developed using actively cooled NIR semiconductor arrays (VIRTIS) or uncooled MIR bolometer arrays. The performance of the opto-electronical imaging spectrometers results in high spatial and radiometric resolution at moderate spectral resolution. Simultaneous observations at high spectral resolutions were realized either by integration of an additional high spectral resolution infrared cross-dispersed spectrometer using prism and grating for dispersion (VIRTIS-H) or by the use of a Fourier Transform Spectrometer (PFS). Space qualification of planetary spectrometers leads to systems having robust and reliable thermo-mechanical/ thermo-electrical properties to survive strong vibrations, thermal stress due to large thermal gradients, and extreme solar radiation fluxes. Challenges in mass, volume, and power consumption make it necessary to adapt light weight systems and miniaturized units like shutters, microradiometers, and electronic units. Finally, the internal instrument operations, operations of interfaces to the spacecraft, and procedures for data exchange with the ground station require qualified onboard software solutions in accordance with different observation modes and onboard calibration procedures.

The observations of spaceborne spectrometers like VIRTIS, MERTIS, and PFS have essentially contributed to answer fundamental questions of research in comparative planetology. Using the spectral information, it succeeded to extract data about the rocks, the rock-forming minerals, and ices on planetary surfaces. These results can be combined with geochronological and geophysical data. This background enables processes of similarities and differences as well as divergent evolutionary paths of the inner planets to be discussed. The Omega VIS/NIR dating of Martian surface features by mineralogical classification is an outstanding example for the capability of this method. It opens an alternative way to the cratering record in determination of planetary surface ages. Combined with future studies of MERTIS on BepiColombo, this method can be applied to Mercury, helping to unveil the poorly known history of origin and evolution of the innermost planet in our solar system. Although the planet Venus has a dense atmosphere being almost opaque in the VIS/IR spectral range, small nightside NIR atmospheric windows enable studies of surface and near surface processes with VIRTIS-M-IR on Venus Express. The extraction of global Venusian temperature and emissivity data makes high demands on simulations of the radiative transfer in the atmosphere. Current improvements to radiative transfer simulations hold a lot of promise for the determination of new datasets for the surface and near surface analyses. The measurements of VIRTIS on ROSETTA are focused on investigations of small bodies in the solar system. Asteroids and comets have more primitive compositions, representing less differentiated material. Studies of minor bodies enable the analysis of the source material forming the larger objects in our solar system. Small bodies in the solar system are enriched in volatiles and may have played an important role in the course of incorporation of volatiles into the material that has formed the terrestrial planets. An important object of research is the water cycle in the planetary system. The detection of water vapor and water ices and the determination of water abundances provide information about the origin of planetary bodies, their atmospheres, and the origin of life. Further developments in planetary spectroscopy for studies of water in the outer solar system aim at an increase of spatial and spectral resolution and improvements of the signal-to- 
noise quality at once. In order to use the $6.25 \mu \mathrm{m}$ water bands for structural ice studies, technical solutions are required to fill the current gap of observations between 5 and $7 \mu \mathrm{m}$. In this way, continuous spectral studies of planetary surfaces and atmospheres will make an important contribution to future comparative planetology research as well.

\section{REFERENCES}

[1] Allen, D.A., "The dark side of Venus", Icarus 69, 221-229 (1987).

[2] Allen, D.A. and Crawford, J.W., "Cloud structure on the dark side of Venus", Nature 307, 222-224 (1984).

[3] Arnold, G.E., "Measurements of the spectral emittance of particulate minerals and some remote sensing implications", Vibrational Spectroscopy 2, 245-249 (1991).

[4] Arnold, G.E. et al., "Studies of Martian atmosphere and surface by the Planetary Fourier Spectrometer on board the Mars-94 mission", Proc. SPIE 2089, 284-285 (1993).

[5] Arnold, G.E. et al., "Investigation of surface properties by the Planetary Fourier Spectrometer (PFS) onboard of the Mars 96 mission", Proc. IAF, \$-Q.3.33, 1-6 (1994).

[6] Arnold, G.E. et al., "MERTIS - Mercury Radiometer and Thermal Infrared Spectrometer- a novel thermal imaging spectrometer for the exploration of Mercury", J. Appl. Rem. Sens. 2, 023528 (2008).

[7] Arnold, G.E. et al., "Venus surface data extraction from VIRTIS/Venus Express measurements: Estimation of a quantitative approach“, J. Geophys. Res. 13, E00B10 (2008).

[8] Arnold G.E. et al., "Thermal infrared imaging of Mercury - MERTIS a new remote sensing technology", Proc. SPIE 7453, 745305-74310 (2009).

[9] Arnold, G.E. et al., "MERTIS-Thermal infrared imaging of Mercury - Advances in mid IR remote sensing technology for planetary exploration", Proc. SPIE 7808, 78081 (2010).

[10] Arnold, G.E. et al., "Venus atmospheric and surface studies from VIRTIS on Venus Express", Proc. SPIE 8154-31, this issue (2011).

[11] Arnold, G.E. et al., "A new perspective on Mercury's surface composition and temperatures - Mercury Radiometer and Thermal Infrared Spectrometer (MERTIS)", Proc. SPIE 8154-32, this issue (2011).

[12] Bauer, M., "MERTIS: geometrical calibration of thermal infrared optical system by applying diffractive optical elements", Proc. SPIE 8154-38, this issue (2011).

[13] Barlow, N.G., "Mars", Cambridge University Press, 102-110 (2008).

[14] Basilevsky, A.T. et al., "The resurfacing history of Venus". In: Venus II: Geology, Geophysics, Atmosphere, and Solar Wind Environment. Bougher, S.W., Hunten, D.M., Phillips, R.J. (Eds.), University of Arizona Press, Tucson, 1047-1086 (1997).

[15] Basilevsky, A.T. and Head, J.W., "The geologic history of Venus: A stratigraphic view”, J. Geophys. Res. 103, 8531-8544 (1998).

[16] Basilevsky, A.T. and Head, J.W., "Geologic units on Venus: Evidence for their global correlation”, Planet. Space Sci. 48, 75-111 (2000).

[17] Basilevsky, A.T. and Head, J.W., "Venus: Timing and rates of geologic activity", Geology 30, 1015-1018 (2002).

[18] Basilevsky, A.T. et al., "Impact crater air fall deposits on the surface of Venus: Areal distribution, estimated thickness, recognition in surface panoramas, and implications for provenance of sampled surface materials", J. Geophys. Res. 109(E12003) (2004).

[19] Basilevsky, A.T. et al., "Landing on Venus: Past and future", Planet. Space Sci. 55, 2097-2112 (2007).

[20] Benz, W. et al., "Collisional stripping of Mercury's mantle”, Icarus 74, 516-528 (1988).

[21] Benz, W. et al., "The origin of Mercury", Space Sci. Rev. 132, 189-202 (2007).

[22] Bézard, B. et al., "Water vapor abundance near the surface of Venus from Venus Express / VIRTIS observations", J. Geophys. Res. 114(E00B39) (2009).

[23] Bibring, J.-P. et al., "Mars surface diversity as revealed by the OMEGA/Mars Express observations", Science 11, 1576-1581 (2005).

[24] Blewett, D.T., et al., "Multispectral images of Mercury from the first MESSENGER flyby: Analysis of global and regional color trends", Earth Planet. Sci. Lett. 285, 272-282 (2009).

[25] Brown, S. and Elkins-Tanton, L.T., "Composition of Mercury's oldest crust from magmaocean models", Earth and Planet. Sci. Lett. 286, 446-455 (2009).

[26] Burbine, T.H. et al., "Spectra of extremely reduced assemblages: Implications to Mercury“, Metoritics \& Planet. Sci. 37, 1233-1244 (2002). 
[27] Burns, R.G., "Origin of electronic spectra of minerals in the visible to near-infrared region". In: Remote Geochemical Analysis: Elemental and Mineralogical Composition. Pieters, C.M., Englert, P.A.J. (Eds.), Cambridge Univ. Press, New York, pp. 3-29 (1993).

[28] Cameron, A.G.W., "The partial volatilization of Mercury", Icarus 64, 285-294 (1985).

[29] Cameron, A.G.W. et al., "The strange density of Mercury: Theoretical considerations". In: Mercury. Matthews M.S., Chapman, C., and Vilas, F. (Eds.), University of Arizona Press, Tucson, AZ, 692-708 (1988).

[30] Chambers, J.E., "Planetesimal formation by turbulent concentration", Icarus 208, 505-517 (2010).

[31] Christensen, P.R. et al., "Thermal emission spectrometer experiment - Mars Observer Mission", J. Geophys. Res. 97(E5), 7719-7734 (1992).

[32] Christensen, P.R. et al., "The Thermal Emission Imaging System (THEMIS) for the Mars 2001 Odyssey Mission", Space Sci. Rev. 110, 85-130 (2004).

[33] Clark, B.E. et al., "E-type asteroid spectroscopy and compositional modeling", J. Geophys. Res. 109, E02001 (2004).

[34] Coradini, A. et al., "VIRTIS: An imaging spectrometer for the Rosetta mission", Planet. Space Sci. 46, 1291-1304 (1998).

[35] Coradini, A. et al.., "VIRTIS: An imaging spectrometer of the Rosetta mission," Space Science Rev. 128, 529-559 (2007).

[36] Coradini, A. et al., "VIRTIS: An imaging spectrometer for the Rosetta mission," In: Rosetta: ESA's Mission to the Origin of the Solar System, Springer, 565-587 (2009).

[37] Coradini, A. et al., "The surface composition and temperature of asteroid 21 Lutetia as observed by Rosetta/VIRTIS", submitted to Science, (2011).

[38] Denevi, B.W. et al., "The evolution of Mercury's crust: A global perspective from MESSENGER”, Science 324, 613-618 (2009).

[39] DeSanctis, M.C. et al., "Shape and obliquity effects on the thermal evolution of the Rosetta target 67P/ChuryumovGerasimenko cometary nucleus", Icarus 207, 341-358 (2010).

[40] Drossart, P. et al., "Scientific goals for the observation of Venus by VIRTIS on ESA/Venus Express mission", Planet. Space Sci. 55, 1653-1672 (2007).

[41] Ford, J.P. et al., "Guide to Magellan image interpretation“, Pasadena, JPL 93-24 (1993).

[42] Formisano, V. et al., "The Planetary Fourier Spectrometer (PFS) for Mars Epress". In: The Mars Express mission of ESA, ESA SP (2004).

[43] Formisano, V. et al., "The Planetary Fourier Spectrometer (PFS) onboard the European Mars Express mission", Planet. Space. Sci. 53, 963-974 (2005).

[44] Gaffey, M.J. and Kelley, M.S., "Mineralogical variations among high albedo E-type asteroids: Implications for asteroid igneous processes", Proc. LPSC XXXV, 1812 (2004).

[45] Hartmann, W.K. and Neukum, G., "Cratering chronology and the evolution of Mars", Space Sci. Rev. 96, 165-194 (2001).

[46] Hashimoto, G.L. and Sugita, S., "On observing the compositional variability of the surface of Venus using nightside near-infrared thermal radiation", J. Geophys. Res. 108(E9), 5109 (2003).

[47] Haus, R. and Arnold, G., "Radiative transfer in the atmosphere of Venus and application to surface emissivity retrieval from VIRTIS/VEX measurements", Planet. Space Sci. 58, 1578-1598 (2010).

[48] Head, J.W. et al., "Venus volcanism: Classification of volcanic features and structures, associations, and global distribution from Magellan data", J. Geophys. Res. 97, 13153-13197 (1992).

[49] Head, J.W. et al., "Evidence for intrusive activity on Mercury from the first MESSENGER flyby", Earth Planet. Sci. Lett. 285, 251-262 (2009).

[50] Head, J.W. et al., "Volcanism on Mercury: Evidence from the first MESSENGER flyby for extrusive and explosive activity and the volcanic origin of plains", Earth Planet. Sci. Lett. 285, 227-242 (2009).

[51] Helbert, J. et al., "Surface brightness variations seen by VIRTIS on Venus Express and implications for the evolution of the Lada Terra region", Geophys. Res. Lett. 35(L11201) (2008).

[52] Helbert, J. and Maturilli, A., "The emissivity of fine-grained labradorite sample at typical Mercury dayside temperatures", Earth and Planet. Sci. Lett. 285, 347-354 (2009).

[53] Hiesinger, H. and J. Helbert, "The Mercury Radiometer and Thermal Infrared Spectrometer (MERTIS) for the Bepi Colombo Mission”, Planet. Space Sci. 58, 144-165 (2010).

[54] Hirsch, H. and Arnold, G., "Fourier Transform Spectroscopy in remote sensing of solid planetary surfaces", Vib. Spectrosc. 5, 119-123 (1993). 
[55] Hirsch, H. et al., "The optical definition of the Planetary Fourier Spectrometer (PFS): An FTIR spectrometer for the Mars '96 mission”, Proc. SPIE 2268, 331 (1994).

[56] Hirsch, H. et al., "The Planetary Fourier Spectrometer (PFS) for the orbiter of the spacecraft Mars 96", Planet. Space Sci. 44, 889-897 (1996).

[57] Kappel, D. et al., "Refinements in the data analysis of VIRTIS-M-IR Venus nightside spectra", submitted to Adv. Space Res. (2011).

[58] Keller, H.U. et al., "E-Type asteroid (2867) Šteins as imaged by OSIRIS on board Rosetta", Science 327, 190-193 (2010).

[59] Kreslavsky, M.A. et al., "Dielectric permittivity of the tessera surface material on Venus inferred from Magellan radar data", Solar System Res. 34, 379-389 (2000).

[60] Küppers, M. et al., "Determination of the light curve of the Rosetta target asteroid (2867) Šteins by the OSIRIS cameras onboard Rosetta", Astronomy and Astrophys. Lett. 462, L13-U3 (2007).

[61] Lamy, P.L. et al., "Multi-color, rotational resolved photometry of asteroid 21 Lutetia from Osiris/Rosetta observations", Astronomy and Astrophys. 521, A19 (2010).

[62] McCoy, T.J. et al., "Partial melting of the Indrach (EH4) meteorite: A textural, chemical, and phase relations view of melting and melt migration", Metoritics \& Planet. Sci. 34, 735-746 (1999).

[63] McKenzie, D. et al., "Pancakelike domes on Venus", J. Geophys. Res. 97, 15967-15976 (1992).

[64] McKinnon, W.B. et al., "Cratering on Venus: Models and observations". In: Venus II: Geology, Geophysics, Atmosphere, and Solar Wind Environment. Bougher, S.W., Hunten, D.M., Phillips, R.J. (Eds.), University of Arizona Press, Tucson, 969-1014 (1997).

[65] Meadows, V.S. and Crisp, D., "Ground-based near-infrared observations of the Venus nightside: The thermal structure and water abundance near the surface", J. Geophys. Res. 101, 4595-4622 (1996).

[66] Morbidelli, A. et al., "Source regions and time scales for delivery of water to Earth", Meteoritics 35, 1309-1320 (2000).

[67] Müller, N. et al., "Venus surface thermal emission at $1 \mu \mathrm{m}$ in VIRTIS imaging observations: Evidence for variation of crust and mantle differentiation conditions", J. Geophys. Res. 113(E00B17) (2008).

[68] Murchie, S.L. et al., "Compact Reconnaissance Imaging Spectrometer for Mars investigation and data set from the Mars Reconnaissance Orbiter's primary science phase”, J. Geophys. Res., 114(E00D07) (2009).

[69] Nikolaeva, O.V. et al., "Evidence of the crustal dichotomy. In: Venus geology, geochemistry, and geophysics Research results from the USSR", Barsukov, V.L. et al. (Eds.), Univ. of Arizona Press, Tucson, 129-139 (1992).

[70] Piccioni, G. et al., "VIRTIS: The Visible and Infrared Thermal Imaging Spectrometer", ESA Spec. Publ. SP-1295, 1-27, European Space Agency, Paris (2007).

[71] Raymond, S.N. et al., "Building the Terrestrial Planets: Constrained Accretion in the Inner Solar System", Icarus 203, 644-662 (2009).

[72] Säuberlich, T. et al., "MERTIS: configuration of measurement sequences for a maximized image SNR", Proc. SPIE 8154-36, this issue (2011).

[73] Schulz, R., "Rosetta-one comet rendezvous and two asteroid fly-bys", Solar System Res. 43, 343-352 (2009).

[74] Schulz, R., "The Rosetta mission and its fly-by at asteroid 2867 Šteins", Planet. Space Sci. 58, 1057 (2010).

[75] Shestopalov, D.I. et al., "Titanium-bearing pyroxenes of some E asteroids: Coexisting of igneous and hydrated rocks", Planet. Space Sci. 58, 1400-1403 (2010).

[76] Smrekar, S.E. et al., "Recent hot-spot volcanism on Venus from VIRTIS emissivity data", Science 328, 605- 608 (2010).

[77] Sprague, A.L. et al., "Evidence for anorthosite and basalt from mid-infrared (7.3-13.5 micron) spectroscopy", Icarus 109, 156-167 (1994).

[78] Sprague, A.L. et al., "Sulfur at Mercury, elemental at the poles and sulfides in the regolith", Icarus 118, 211-215 (1995).

[79] Sprague, A.L. et al., "Mercury's feldspar connection: Mid-IR measurements suggest plagioclase”, Adv. Space Res. 19, 1507-1510 (1997).

[80] Sprague, A.L. and Roush, T.L., "Comparison of laboratory emission spectra with Mercury telescopic data”, Icarus 133, 174-183 (1998).

[81] Sprague, A.L. et al., "Mid-infrared (8.1-12.5 $\mu \mathrm{m}$ ) imaging of Mercury", Icarus 147, 421-432 (2000).

[82] Sprague, A. L., "Thermal emission spectroscopy and data analysis of solar system regoliths", ASP Conference 196, 167-186 (2000).

[83] Sprague, A.L. et al., "Mid-infrared (3-13.5 $\mu \mathrm{m})$ observations show heterogeneous composition, presence of 
intermediate and basic soil types, and pyroxene", Meteoritics and Planet. Sci. 37, 1255-1268 (2002).

[84] Sprague, A.L. et al., "Mercury's surface composition and character as measured by ground-based observations", Space Sci. Rev. 132, 399-431 (2007).

[85] Sprague, A.L. et al., "Mercury: Mg-rich mineralogy with K-spar and garnet”, LPSC 39, 1320 (2008).

[86] Sprague, A.L. et al., "Spectral emissivity measurements of Mercury's surface indicate Mg- and Ca-rich mineralogy, K-spar, Na-rich plagioclase, rutile, with possible perovskite, and garnet”, Planet. Space Sci. 57, 364-383 (2009).

[87] Strom, R.G. and Sprague, A.L., "Exploring Mercury: The iron planet", Springer Praxis, Chichester UK, p. 216 (2003).

[88] Strom, R.G. et al., "Mercury crater statistics from MESSENGER flybys: Implications for stratigraphy and resurfacing history", Planet. Space Sci., in press (2011).

[89] Tanaka, K.L. et al., "Global stratigraphy”. In: Mars. Kieffer, H.H. et al. (Eds.), Univ. of Arizona Press, 345-382 (1992).

[90] Taylor, F.W., "Venus before Venus Express", Planet. Space Sci. 54, 1249-1262 (2006).

[91] Thomas, N. et al., "Rosetta/OSIRIS observations of the surface geomorphology of 21 Lutetia", Proc. EPSC Abstracts 5, EPSC2010-340 (2010).

[92] Tsang, C.C.C. et al., "A correlated-k model of radiative transfer in the near-infrared windows of Venus", J. Quant. Spectrosc. Radiat. Transfer 109, 1118-1135 (2008),

[93] Walter, I. et al., "Deep space instrument design for thermal infrared imaging with MERTIS, Proc. SPIE 8154-33, this issue (2011).

[94] Warell, J. et al., "The 0.7-5.3 $\mu \mathrm{m}$ IR spectra of Mercury and the Moon: Evidence for high-Ca clinopyroxene on Mercury", Icarus 180, 281-291 (2006).

[95] Warell, J. et al., "Constraints on Mercury's surface composition from MESSENGER and ground-based Spectroscopy", Icarus 209, 138-163 (2010).

[96] Weidenschilling, S. J., "Iron/silicate fractionation and the origin of Mercury", Icarus 35, 99-111 (1978).

[97] Wetherill, G.W., "Accumulation of Mercury from planetisimals". In: Mercury. Matthews M.S., Chapman, C., and Vilas, F. (Eds.), University of Arizona Press, Tucson, AZ, 670-691 (1988).

[98] Zeh, T. et al., "MERTIS: reflective baffle design and manufacturing", Proc. SPIE 7808, 7808-22 (2010). 